\title{
Prostate cancer screening and the role of PSA: A UK perspective
}

$P$ ROSTATE CANCER is the most common solid-organ cancer and the second-leading cause of cancer death in Western men. Nearly 50,000 men are diagnosed with prostate cancer each year in the United Kingdom, and more than 11,000 die of it. ${ }^{2}$ Prostate cancer is therefore a significant killer of men. And it is usually a silent killer, asymptomatic in its curable stages. Hence, to save lives from prostate cancer, we must diagnose it early, before symptoms appear.

See related editorial, page 17

Fortunately, the serum biomarker prostate-

We advocate

PSA screening

for all middle-

aged men,

especially

if they have

risk factors tate cancer (intervention) or not (control). ${ }^{3}$ Screened men had PSA tests every 2 to 4 years and a prostate biopsy if their PSA concentration was greater than $3 \mathrm{ng} / \mathrm{mL}$. At 16 years of follow-up, ${ }^{3} 20 \%$ fewer men had died of prostate cancer in the intervention group than in the control group. The number needed to be screened to diagnose 1 case of prostate cancer was 18 in this latest follow-up of the study, a significant lowering compared with the prior study report.

The study investigators concluded ${ }^{3}$ correctly that PSA screening significantly reduces prostate cancer mortality, with a larger $a b-$ solute benefit with longer follow-up. Hence, my view is that for men with a long life expectancy (ie, most middle-aged men), screening for prostate cancer with PSA is warranted.

\section{The PLCO study provides no useful information over the ERSPC}

The US Prostate, Lung, Colorectal, and Ovarian Cancer Screening Trial (PLCO) ${ }^{4}$ which included a prostate-screening arm, found more cancers in screened men but no survival advantage.

However, the PLCO study was smaller than the ERSPC and was heavily contaminated, as $44 \%$ of men in the control group (assigned to no-screening) had PSA tests anyway, so really it was a study of screening vs less screening. Further, the assigned interventions and subsequent investigations were not well adhered to: some men allocated to having their PSA checked did not get tested, $44 \%$ of men allocated to no-PSA testing got tested anyway, and only about a third of patients with a PSA level higher than $4 \mathrm{ng} / \mathrm{mL}$ had a prostate biopsy. ${ }^{4}$ All in all, this study does not really provide any useful information over ERSPC. 
Active surveillance is now preferred in most men with low-risk prostate cancer Plenty of evidence from several studies shows that low-risk prostate cancers $($ PSA $<10$ $\mathrm{ng} / \mathrm{mL}$, Gleason grade 6, and unilateral cancer) usually grow slowly and are safe to monitor, with active treatment advised if surveillance tests show progression.

The world's largest comparative-effectiveness randomized study of PSA-screened interventions (ProtecT) showed no survival benefit from surgery or radiation therapy compared with active surveillance at a median of 10 years. ${ }^{5}$

\section{Prostate biopsy is no longer always the next step for men with elevated PSA}

In my opinion, multiparametric magnetic resonance imaging (MRI) should be the next step in the investigation of men who have an elevated PSA. This allows men with a normal scan to be monitored, since MRI will detect most clinically significant prostate cancers (negative predictive value 80\%-90\%). ${ }^{6}$ Men with suspicious findings on MRI can proceed to prostate biopsy.

While this has become widespread practice in the United Kingdom, in the United States many insurance companies will not reimburse for prebiopsy MRI, and thus, alternatives such as blood-based biomarkers are often used. There are no head-to-head studies comparing prebiopsy biomarkers and MRI; however, MRI can be used to guide the locations of any subsequent biopsy (see below), whereas biomarkers cannot. I prefer to use MRI.

\section{Prostate biopsy is more accurate and has fewer side effects than ever before} Many prostate cancer experts have replaced transrectal prostate biopsy with MRI-targeted transperineal template biopsy, performed as an outpatient procedure with the patient under general anesthetic. As well as enhancing the prostate cancer detection rate, this technique also reduces the risk of biopsy-related infections and thus decreases antibiotic resistance. Further, fusing the prebiopsy MRI images onto the biopsy platform improves the accuracy of targeting suspicious lesions on MRI; these "fusion" biopsies improve detection of clinically significant cancer while decreasing detection of indolent disease. ${ }^{?}$

Again, although this technique is gaining in popularity in the United Kingdom, it is significantly more expensive than prostate biopsy under local anesthesia, and thus has had limited uptake so far in the United States.

\section{PSA level before age 50 accurately predicts future risk of prostate cancer}

Several studies have shown that the PSA level before age 50 is a stronger predictor of prostate cancer risk than race or family history. ${ }^{8}$ This information could be used to guide the frequency of future PSA testing: "smart" screening. ${ }^{9}$ A 45 -year-old man with a PSA level less than $1 \mathrm{ng} / \mathrm{mL}$ would be advised that his next PSA test should be done in 5 years' time, whereas a man of the same age and race with the same family history with a PSA of 1.5 $\mathrm{ng} / \mathrm{mL}$ would be advised to have it rechecked in a year.

Further, incorporating novel biomarker panels such as the $4 \mathrm{~K}$ score, PSA derivatives like PSA density, and polygenic risk scores can improve the accuracy of prostate cancer screening and give more confidence in determining which men to investigate further, which to monitor and at what frequency, and which to safely discharge.

\section{KEY POINTS}

- Prostate cancer is a significant killer of men.

- Prostate cancer is asymptomatic during its curable stages.

- PSA screening saves lives.

- Patients with low-risk prostate cancer do not generally need treatment, whereas those with intermediate- and high-risk cancers usually benefit from curative therapy.

- Not all men with a raised PSA need a prostate biopsy, thanks to MRI scanning.

- Prostate biopsy is now more accurate, safer, and more comfortable for patients when informed by an MRI.

- PSA levels before age 50 accurately predict future risk of developing prostate cancer.

\section{DISCLOSURES}

The author reports no relevant financial relationships which, in the context of their contributions, could be perceived as a potential conflict of interest.
Patients with

low-risk

prostate

cancer do not

generally

need treatment 


\section{REFERENCES}

1. Siegel RL, Miller KD, Jemal A. Cancer statistics, 2020. CA Cancer J Clin 2020; 70(1):7-30. doi:10.3322/caac.21590

2. Prostate Cancer UK. About prostate cancer. https://pros tatecanceruk.org/prostate-information/about-prostatecancer. Accessed November 30, 2020.

3. Hugosson J, Roobol MJ, Mansson M, et al. A 16-yr follow-up of the European Randomised Study of Screening for Prostate Cancer. Eur Urol 2019; 76(1);45-51. doi:10.1016/j.eururo.2019.02.009

4. Pinsky PF, Prorok PC, Yu K, Kramer BS, Black A. Extended mortality results for prostate cancer screening in the PLCO trial with median follow-up of 15 years. Cancer 2017; 123(4):592-599. doi:10.1002/cncr.30474

5. Hamdy FC, Donovan JL, Lane JA, et al. 10-year outcomes after monitoring, surgery, or radiotherapy for localized prostate cancer. N Engl J Med 2016; 375(15):1415-1424. doi:10.1056/NEJMoa1606220

6. Bryant RJ, Hobbs CP, Eyre KS, et al. Comparison of pros tate biopsy with or without multiparametric magnetic resonance imaging for prostate cancer detection: an observational cohort study. J Urol 2019; 201(3):510-519. doi:10.1016/j.juro.2018.09.049

7. Kasivisvanathan V, Stabile A, Neves JB, et al. Magnetic resonance imaging-targeted biopsy versus systematic biopsy in the detection of prostate cancer: a systematic review and meta-analysis. Eur Urol 2019; 76(3):284-303. doi:10.1016/j.eururo.2019.04.043

8. Lilja H, Cronin AM, Dahlin A, et al. Prediction of significant prostate cancer diagnosed 20 to 30 years later with a single measure of prostate-specific antigen at or before age 50. Cancer 2011; 117(6):1210-1219. doi:10.1002/cncr. 25568

9. Sooriakumaran P. Smarter screening for prostate cancer: for the few, not the many? A stratified approach based on baseline risk. Expert Rev Anticancer Ther 2011: 11(2):169-172. doi:10.1586/era.10.233

Address: Prasanna Sooriakumaran, MD, PhD, FRCSUrol, FEBU, Digestive Diseases and Surgery Institute, Cleveland Clinic Lon don, 33 Grosvenor Pl, Belgravia, London SW1X 7HY, United

Kingdom; psoori@santishealth.org 